\title{
Platelet-Rich Plasma Injection in Patients with Atrophic Rhinitis
}

\author{
Do Hyun Kim Min Hyeong Lee Jaeyoon Lee Eun A. Song \\ Soo Whan Kim Sung Won Kim \\ Department of Otolaryngology-Head and Neck Surgery, Seoul St. Mary's Hospital, College of \\ Medicine, The Catholic University of Korea, Seoul, Republic of Korea
}

\section{Keywords}

Platelet-rich plasma · Atrophic rhinitis · Age factors

\begin{abstract}
Objective: To investigate the effect of platelet-rich plasma (PRP) injection in patients with atrophic rhinitis. Methods: Prepared PRP was injected into the inferior turbinate bilaterally, and nasal bacterial cultures were conducted. Improvement of symptoms was assessed with the Nasal Obstruction Symptom Evaluation (NOSE) and the Sino-Nasal Outcome Test-22 (SNOT-22). Nasal mucociliary clearance was assessed using the saccharin transit time (STT). Results: In the PRPinjected group (group A), NOSE (throughout the study) and SNOT-22 (1 month after injection) scores were significantly decreased during the study. However, the saline spray group (group B) showed no significant nasal symptom improvement during the study period. In group A, the STT was improved until 3 months after the injection. In contrast, group B showed STT improvement after 2 months that was maintained throughout the study. Conclusion: PRP injections can improve nasal symptoms and nasal mucociliary function in patients with atrophic rhinitis.

(C) 2021 The Author(s)
\end{abstract} Published by S. Karger AG, Basel

\section{karger@karger.com www.karger.com/orl

\section{Introduction}

Atrophic rhinitis is a chronic condition characterized by progressive atrophy of the nasal mucosa and underlying bone. It leads to the formation of nasal crusts, fetor, nasal obstruction, epistaxis, olfactory disturbances, secondary infection, nasal deformity, and, rarely, intracranial spread [1]. Atrophic rhinitis can be classified as either primary or secondary to a predisposing condition or event. Primary atrophic rhinitis is rare and appears to be more common in patients of low socioeconomic status who have a poor diet and possible iron deficiency or who live in unsanitary conditions [2]. Microbiological investigation usually detects Klebsiella ozaenae. With improvements in hygiene, the incidence of primary atrophic rhinitis has decreased. In contrast, secondary atrophic rhinitis has increased in prevalence due in part to the increase in the older population; the condition in this population is referred to as "geriatric rhinitis" [3]. Another important cause is the increase in turbinate surgery [4], which leads to symptoms that appear several years post-op, can persist throughout the patient's life, and may even overlap with geriatric rhinitis.

Platelet-rich plasma (PRP) was first introduced in 1987 for open heart surgeries. Its use helps repair injured cells using growth and differentiation factors. PRP is now 
widely accepted as an effective means of promoting wound healing and tissue regeneration [5]. Autologous PRP extraction and injection is not associated with allergic reactions or other problems caused by isoantigens or heteroantigens. Recently, the use of PRP has been introduced to support regeneration of nasal mucosa in atrophic rhinitis patients $[6,7]$. Therefore, in this study, we investigated the effect of PRP injection in patients with geriatric rhinitis or secondary atrophic rhinitis caused by turbinoplasty.

\section{Materials and Methods}

\section{Study Design and Patients}

This clinical trial was approved by the Institutional Review Board of the Seoul St. Mary's Hospital (KC16CISI0726). The trial was registered in the database of clinical trials (NCT03112330). This study was monitored by the Catholic Institute of Cell Therapy, Catholic Medical Center, Korea. All investigators conducted this study in accordance with the Declaration of Helsinki. Written informed consent was obtained from all patients before recruitment.

This was a prospective study. Only adult patients (age $>19$ years) with geriatric rhinitis or secondary atrophic rhinitis as a result of turbinoplasty were included in the study. Atrophic rhinitis including geriatric rhinitis was defined as atrophic dry, crusted, irritated nasal mucosa and/or thick mucus on endoscopy with symptoms such as postnasal drainage, nasal obstruction, epistaxis, olfactory changes, or a foul odor. Patients with atrophic rhinitis symptoms and a history of nasal surgery such as turbinoplasty were classified as secondary atrophic rhinitis patients and, otherwise, geriatric rhinitis patients in this study. The following patients were excluded from the study: (1) patients with any bleeding disorder including thrombocytopenia $(<100,000 / \mu \mathrm{L}) ;(2)$ patients with hematologic disorders; (3) patients with nasal polyps, tumors, or sinusitis; (4) patients with active septicemia; (5) patients with diabetes mellitus or uncontrolled hypertension; (6) patients taking anticoagulants; and (7) patients with genetic disorders characterized by ciliary dysfunction. After the exclusion criteria were applied, 26 patients were enrolled in this study between June 08, 2017, and April 01, 2019. These patients were negative for allergies and had no other underlying diseases. Group A was the PRP injection group, and group B was the conventional saline spray treatment group. Patients were randomly assigned to each group using block randomization with a block size of 2. One patient in group $\mathrm{A}$ and 3 patients in group $\mathrm{B}$ were removed from the study due to being lost to follow-up. Therefore, a total of $22 \mathrm{pa}-$ tients (12 in group A; 10 in group B) completed this study. All patients did not use any other topical treatment during the study period.

\section{PRP Preparation and Injection Method}

Twenty-two milliliters of autologous blood were drawn from each patient into a $30-\mathrm{cc}$ syringe containing sodium citrate $3 \%$ as an anticoagulant and shaken gently to mix. The process was performed with a Prosys 30-cc syringe and a PRP centrifuge (PRODIZEN, Seoul, South Korea). Whole blood was centrifuged in 2 ses-

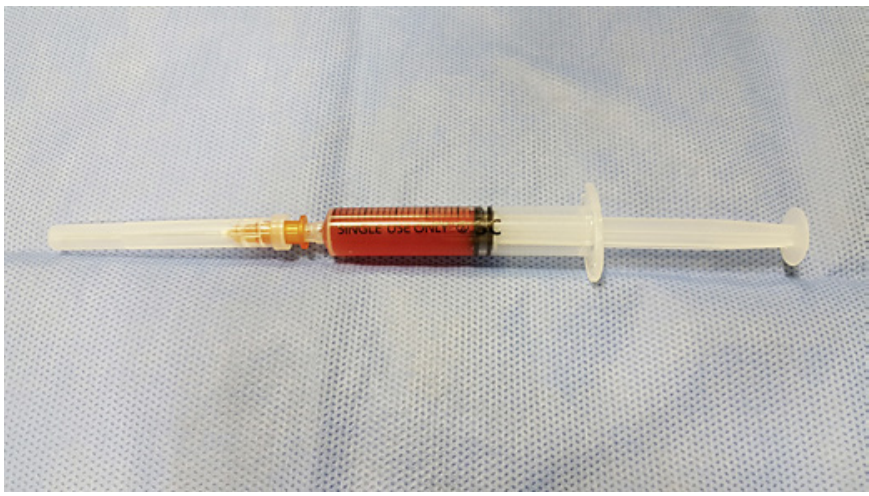

Fig. 1. Prepared platelet-rich plasma injection: about $2-2.5-\mathrm{cc}$ platelet-rich plasma was transferred into a sterile tube using a 5-cc syringe equipped with a 26-gauge needle.

Table 1. Bacterial culture results in the nasal cavity

\begin{tabular}{lll}
\hline & \multicolumn{2}{l}{ Group } \\
\cline { 2 - 3 } & A & B \\
\hline Staphylococcus, coagulase-negative & 1 & 2 \\
Staphylococcus epidermidis & 2 & 1 \\
Streptococcus viridans, alpha-hem & 2 & 0 \\
Bacillus sp. & 1 & 0 \\
Staphylococcus aureus & 0 & 1 \\
Culture negative & 7 & 6 \\
\hline
\end{tabular}

sions. The first was called a soft spin and allowed the blood to be separated into 3 layers: the bottom RBC layer, the top acellular plasma (platelet-poor plasma [PPP]) layer, and the intermediate PRP layer called the buffy coat. The soft spin was conducted for $3 \mathrm{~min}$ at 3,000 speed/RCF for men's blood and 2,800 speed/RCF for women's blood. The PPP layer and buffy coat were transferred into another tube which underwent a second faster centrifugation called a hard spin ( $3 \mathrm{~min}$ at 3,300 speed/RCF). This spin allowed the platelets to settle at the bottom along with a few RBCs. About $2-2.5 \mathrm{cc}$ of the PRP was transferred into a sterile tube using a 5-cc syringe equipped with a 26-gauge needle (Fig. 1). The prepared syringe was sent immediately to the endoscopy room, where the PRP was injected into both anterior portions of the inferior turbinates and the most shrunken area bilaterally (Fig. 2).

\section{Normal Saline Spray Usage Instructions}

Standard buffered isotonic saline spray (Nasaline; Kolmar Korea, Korea) was used. The patients were instructed to use 2 puffs at a time 2 times a day bilaterally during the study. They were instructed not to use the saline spray $3 \mathrm{~h}$ before visiting the hospital to avoid affecting the saccharin test results.

\section{Outcome Measurements}

A nasal cavity bacterial culture was conducted using a Copan $\mathrm{ESwab}^{\mathrm{TM}}$ on the first day of enrollment (Copan Diagnostics, Inc., 

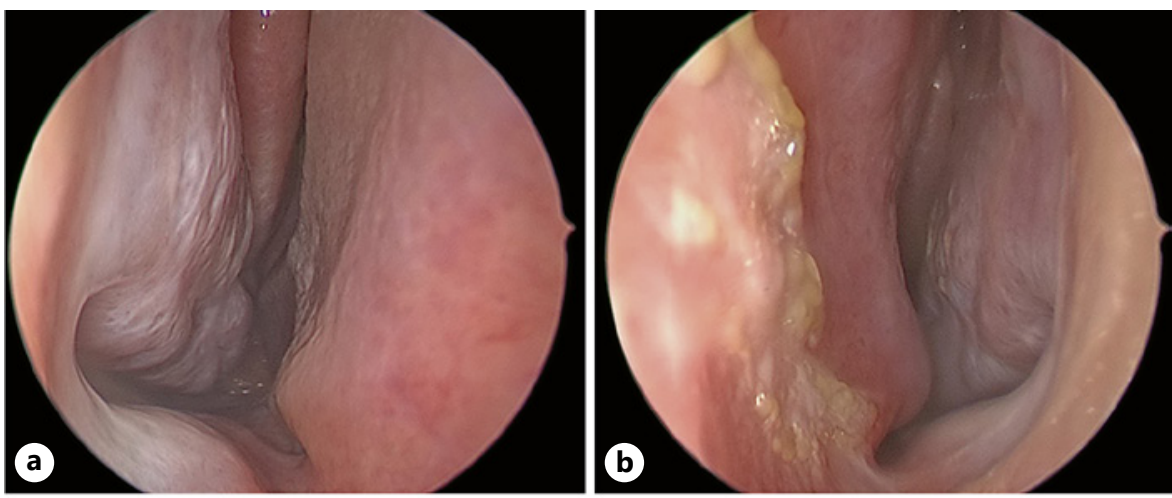

Fig. 2. Platelet-rich plasma injection into the anterior portion of the inferior turbinate: before injection (right nasal cavity; left nasal cavity) (a, b) and during injection (right nasal cavity; left nasal cavity) (c, d).
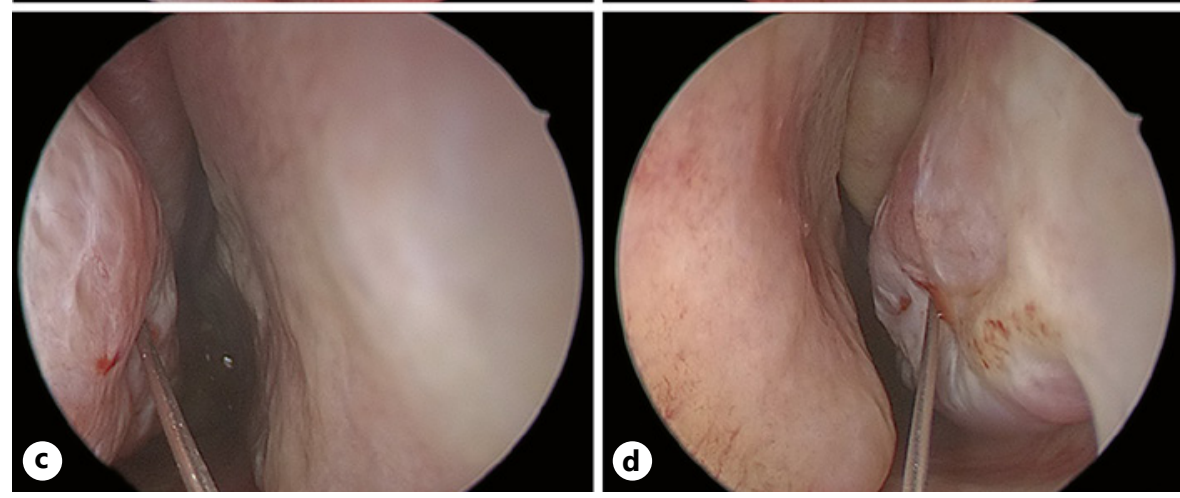

Murrieta, CA, USA). Symptom improvement during the study was assessed using the Nasal Obstruction Symptom Evaluation (NOSE; total scores ranged from 0 to 100) [8] and the Sino-Nasal Outcome Test-22 (SNOT-22) [9]. Symptom questionnaires were distributed to patients on the enrollment day and subsequently at $2,4,8,12$, and 24 weeks after enrollment.

Nasal mucociliary clearance was assessed using the saccharin transit time (STT). A uniformly produced 1-mm particle of sodium saccharine (JMC Corp., Seoul, South Korea) was placed on the surface of the inferior turbinate, $1 \mathrm{~cm}$ behind the head to avoid the area of squamous epithelium [10]. The participants remained seated with their head tipped slightly forward while breathing normally without sneezing or blowing their nose and without taking any substances that might interfere with the test. They were told to indicate when they noted any particular taste. The actual taste they were to expect was not specified in order to avoid false positives. The saccharine particle was carried by means of ciliary transport along the entire nostril until it reached the oropharynx, whereupon a characteristic sweet taste could be perceived. The time elapsed was recorded to the nearest minute, at which time the test was considered complete. If the participant did not detect any taste after $60 \mathrm{~min}$, a saccharine particle was placed on the tongue to ensure that the patient did not suffer any taste abnormalities. The most patent nostril with the least resistance to physiological airflow was chosen.

\section{Statistical Analysis}

All data are expressed as means \pm SD. Paired Student's $t$ test or Wilcoxon's signed-rank test was used to compare pre- and postoperative changes. Fisher's extract test was conducted to calculate proportions differences between the variables. A $p$ value of $<0.05$ was considered to indicate statistical significance. All statistical analyses were conducted using PASW software (PASW Statistics 24; SPSS Inc., Chicago, IL, USA).

\section{Results and Analysis}

The mean age of the enrolled patients was $53.3 \pm 12.1$ (group A, $52.9 \pm 13.4$; group B, $53.8 \pm 11.0$ ) years, and there were 19 males and 3 females (group $A, M / F=11 / 1$; group $\mathrm{B}, \mathrm{M} / \mathrm{F}=10 / 2)$. There were no age $(p=0.870)$, sex $(p=0.571)$, and type of rhinitis $(p=1.000)$ differences between the 2 groups. The nasal cavity bacterial culture results are shown in Table 1 . Only normal nasal cavity flora was detected, with no evidence of Klebsiella ozaenae that could lead to primary atrophic rhinitis.

In group $\mathrm{A}$, the NOSE score decreased significantly throughout the study period (pre vs. $2,4,8,12$, and 24 weeks: $p=0.007,0.004,0.01,0.003$, and 0.009 , respectively). The SNOT-22 scores decreased statistically from 1 month after injection (pre vs. 2, 4, 8, 12, and 24 weeks: $p=0.109,0.002,0.004,0.001$, and 0.002, respectively). However, group B showed no significant improvement in nasal symptoms during the study period (NOSE, pre vs. 

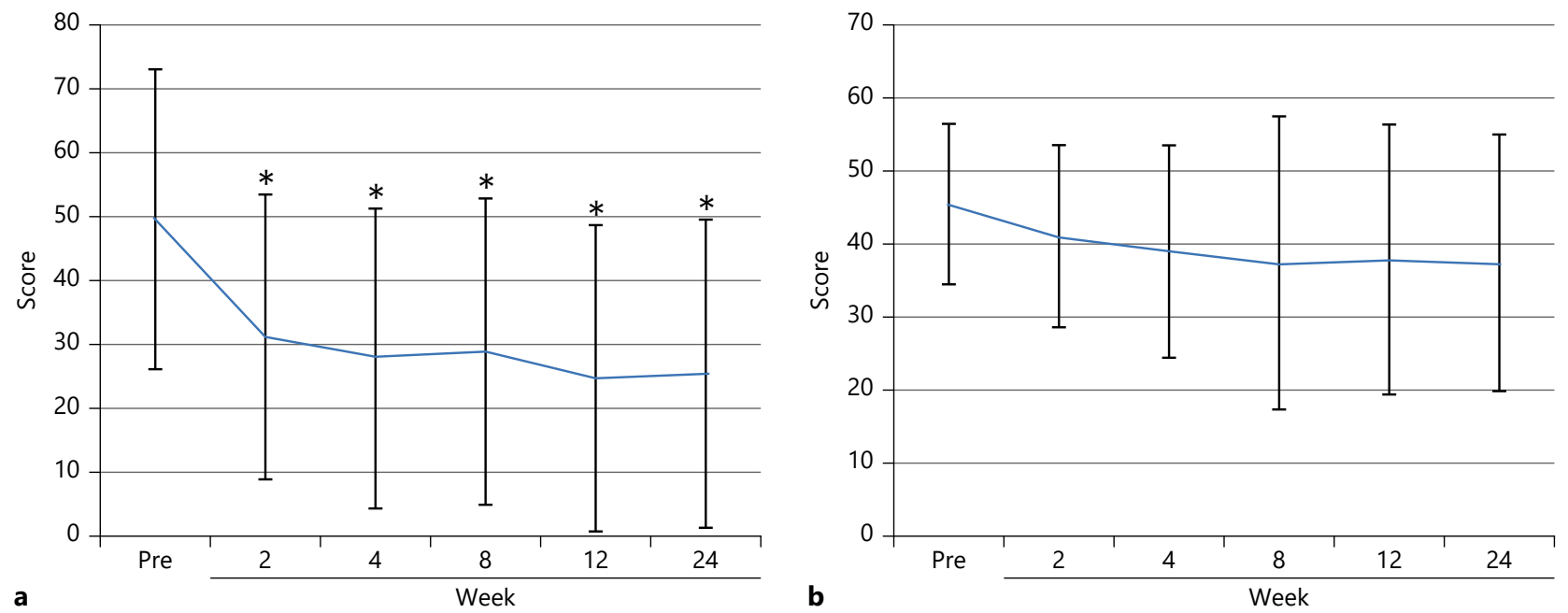

Fig. 3. Nasal Obstruction Symptom Evaluation scores during the study period: group A (a); group B (b). Error bars represent standard deviations. ${ }^{*} p<0.05$, pre versus at each follow-up week.
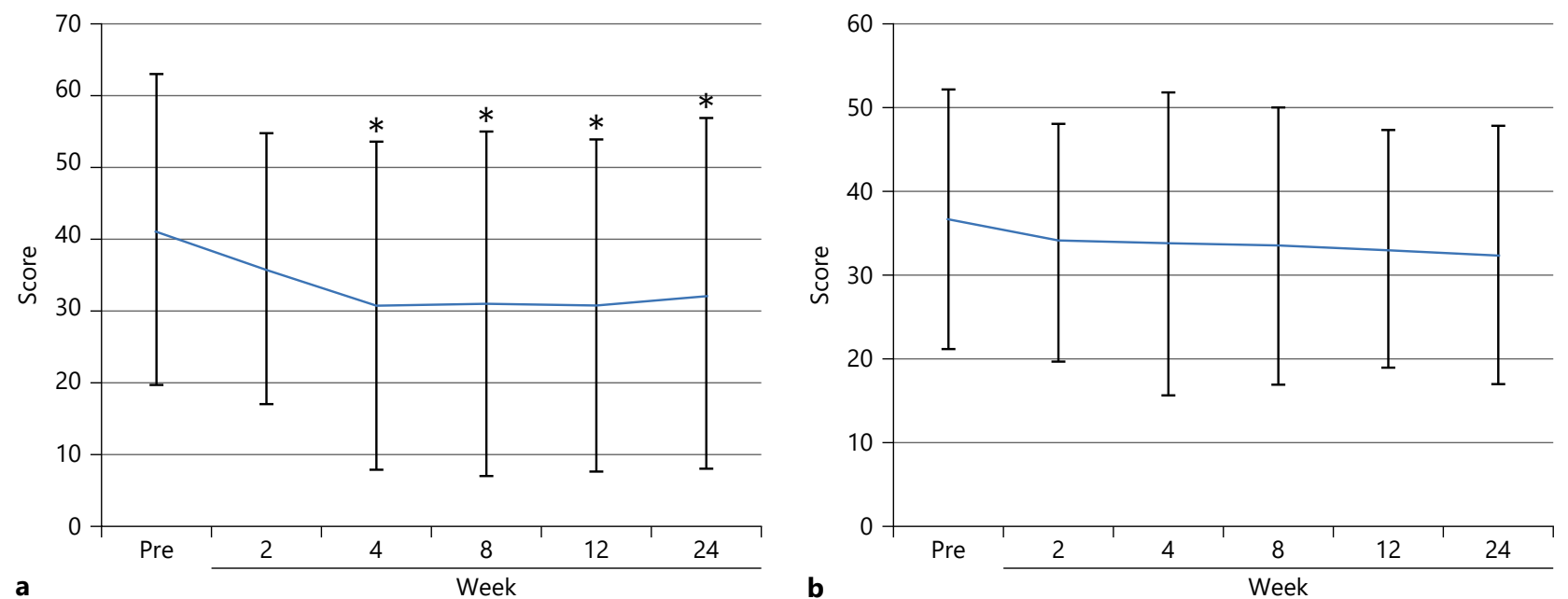

Fig. 4. Sino-Nasal Outcome Test-22 scores during the study period: group A (a); group B (b). Error bars represent standard deviations. ${ }^{*} p<0.05$, pre versus at each follow-up week.

$2,4,8,12$, and 24 weeks: $p=0.215,0.064,0.098,0.086$, and 0.057 , respectively; SNOT-22, pre vs. 2, 4, 8, 12, and 24 weeks: $p=0.123,0.170,0.122,0.082$, and 0.053 , respectively). The NOSE and SNOT-22 results are presented in Figures 3 and 4.

SNOT-22 is categorized into nasal, otologic/facial, sleep, and emotional subdomains [11]. Therefore, sub- analysis was conducted according to subdomains. In group A, nasal, otologic/facial, and sleep subdomains had shown similar statistical results compared to total SNOT-22 scores. However, emotional subdomain showed no statistically significant differences through the study period. Group B also showed no significant differences through the study period similar to total SNOT-22 scores (Table 2). 


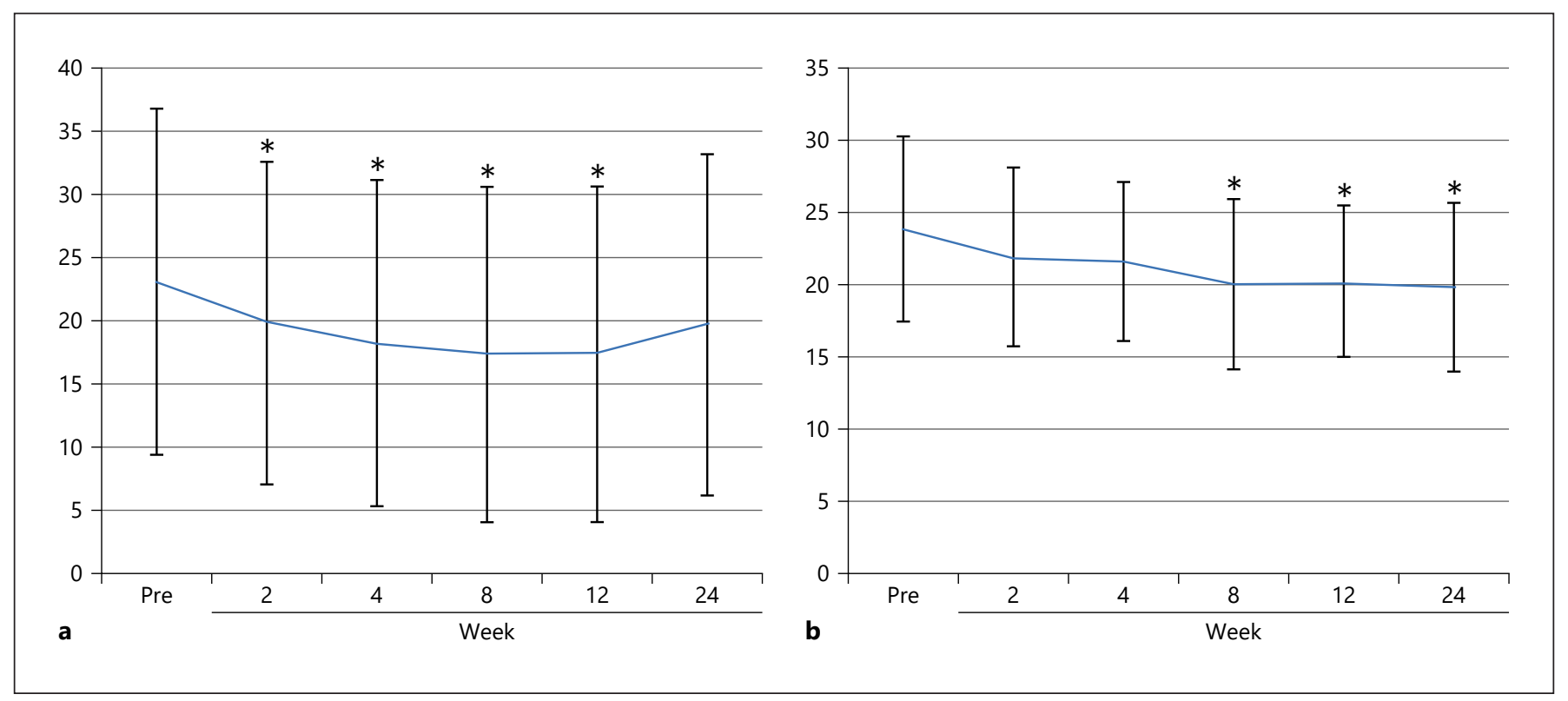

Fig. 5. Saccharin transit time changes during the study period: group A (a); group B (b). Error bars represent standard deviations. ${ }^{*} p<0.05$, pre versus at each follow-up week.

Table 2. Subanalysis according to SNOT-22 subdomains

\begin{tabular}{|c|c|c|c|c|c|c|c|}
\hline \multirow[t]{4}{*}{ A } & Nasal & $13.6 \pm 7.4$ & $11.3 \pm 6.4$ & $8.7 \pm 8.6^{*}$ & $9.1 \pm 8.8^{*}$ & $8.9 \pm 8.3^{*}$ & $9.8 \pm 8.7^{*}$ \\
\hline & Otologic/facial & $4.4 \pm 4.9$ & $3.4 \pm 3.7$ & $2.9 \pm 3.8^{*}$ & $2.8 \pm 3.6^{*}$ & $3.0 \pm 3.5^{*}$ & $3.5 \pm 4.7^{*}$ \\
\hline & Sleep & $20.0 \pm 11.6$ & $17.8 \pm 9.8$ & $16.7 \pm 11.4^{*}$ & $16.4 \pm 12.2^{*}$ & $16.4 \pm 11.9^{*}$ & $16.3 \pm 11.7^{*}$ \\
\hline & Emotional & $3.4 \pm 3.5$ & $3.3 \pm 3.1$ & $2.5 \pm 2.9$ & $2.7 \pm 3.0$ & $2.4 \pm 2.8$ & $2.7 \pm 3.0$ \\
\hline \multirow[t]{2}{*}{ B } & Nasal & $15.4 \pm 6.3$ & $14.4 \pm 5.8$ & $13.5 \pm 7.2$ & $13.8 \pm 7.1$ & $14.1 \pm 6.3$ & $12.4 \pm 5.9$ \\
\hline & Emotional & $2.5 \pm 2.7$ & $2.2 \pm 1.5$ & $2.0 \pm 1.7$ & $2.0 \pm 1.7$ & $1.9 \pm 1.5$ & $1.6 \pm 1.8$ \\
\hline
\end{tabular}

SNOT-22, Sino-Nasal Outcome Test-22. ${ }^{*} p<0.05$, pre versus at each follow-up week.

The STT was improved until 3 months after injection in group A (pre vs. 2, 4, 8, 12, and 24 weeks: $p=0.010$, $0.003,0.005,0.002$, and 0.141 , respectively). Group B showed STT improvement after 2 months (pre vs. 2, 4, 8 , 12 , and 24 weeks: $p=0.088,0.051,0.001,0.001$, and 0.001 , respectively). The STT results are shown in Figure 5.

Subanalysis was performed in group A (Fig. 6), which was divided into subgroups according to age. The younger subgroup (mean age $42.3 \pm 6.3 ; n=6$ ) showed more obvious improved results in nose-related symptoms despite the small numbers (NOSE, pre vs. 2, 4, 8, 12, and 24 weeks: $p=0.027,0.027,0.027,0.027$, and 0.039 , respec- tively; SNOT-22, pre vs. $2,4,8,12$, and 24 weeks: $p=$ $0.249,0.028,0.028,0.027$, and 0.028 , respectively). In contrast, the older subgroup (mean age: $63.5 \pm 9.4 ; n=6$ ) showed relatively poor nasal symptom results (NOSE, pre vs. $2,4,8,12$, and 24 weeks: $p=0.131,0.144,0.197,0.104$, and 0.104 , respectively; SNOT-22, pre vs. $2,4,8,12$, and 24 weeks: $p=0.042,0.080,0.080,0.080$, and 0.080 , respectively). The STT test showed heterogeneous results due to small numbers (younger group, pre vs. $2,4,8,12$, and 24 weeks: $p=0.084,0.026,0.027,0.027$, and 0.058 , respectively; older group, pre vs. $2,4,8,12$, and 24 weeks: $p=$ $0.043,0.043,0.072,0.028$, and 0.249 , respectively). 

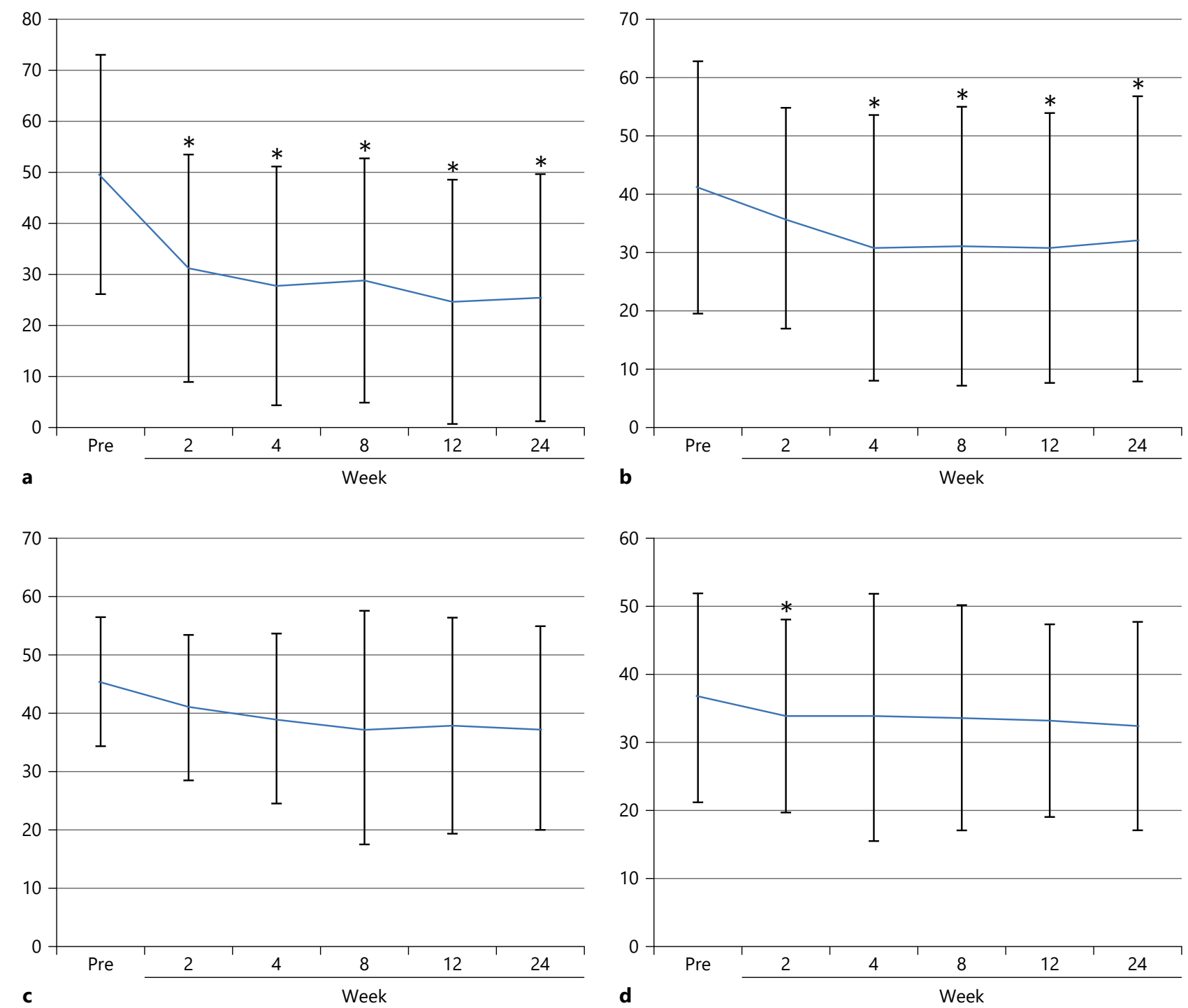

Fig. 6. Group A subanalysis in nasal symptom changes. Subgroups were assigned according to age. NOSE scores in the younger group (a); SNOT-22 scores in the younger group (b); NOSE scores in the older group (c); SNOT22 scores in the older group (d). NOSE, Nasal Obstruction Symptom Evaluation; SNOT-22, Sino-Nasal Outcome Test-22.

\section{Discussion}

The treatment of atrophic rhinitis has 4 main approaches: reduce the nasal cavity with various substances and implants, promote the regeneration of normal mucosa using a classical operation or modified Young's operation [1], lubricate the nasal mucosa, or improve the vascularity of the nasal cavity [1]. Many other treatment approaches have been attempted, including nasal irriga- tion and flushing, glucose-glycerine nasal drops, liquid paraffin, estradiol in arachis oil, anti-ozaena solution, antibiotics, iron, zinc, protein, vitamin supplements, vasodilators, prostheses, vaccines, placental extract, or acetylcholine with or without pilocarpine. However, these methods have varied effectiveness [1]. In the clinic, nasal irrigation with nasal spray is the most common method to manage atrophic rhinitis symptoms, as it hydrates the nasal mucosa and prevents crust formation [12]. 
Of the above methods, the modified Young's procedure has been shown to be an effective and long-lasting treatment for atrophic rhinitis [13]. However, the resulting open-mouth breathing can cause considerable discomfort for the patient [13]. Lubricants and supplements have been shown to have only limited and short-term effects. Therefore, alternative methods to promote the regeneration of nasal mucosa or vascularization have been investigated.

PRP consists of plasma concentrations in excess of platelet concentrations found in the whole blood. PRP elevated the factors affecting tissue growth, differentiation, and scar healing such as platelet-derived growth factor, transforming growth factor, fibroblast growth factor, endothelial growth factor, and insulin-like growth factor. Therefore, PRP has been proven to have acceptably positive results for various clinical studies with effectively promoting wound healing and tissue regeneration including otolaryngologic field [14]. More specifically, there have been reports that PRP is effective in tympanic membrane, vocal fold and facial nerve regeneration, and healing improvement after tympanoplasty or endoscopic sinus surgery [14]. Also, a pilot study was conducted several years ago to treat atrophic rhinitis with a PRP lipid mixture injection [7]. In addition, PRP uses autologous blood, has no allergy or immune rejection reactions, and can be easily prepared in minutes through 2 centrifuge processes.

In this study, we investigated PRP injection into the atrophic nasal mucosa, which improved mucociliary clearance and improved patients' symptoms during 6 months of follow-up, especially in younger patients, in whom results were more pronounced than in the older group. In lots of atrophic rhinitis cases including geriatric rhinitis, the mucus secretion decreased. Therefore, mucus property became thickened and nasal mucociliary clearance time was delayed. Moisture supplement via saline spray could affect the property of thick mucus and nasal mucociliary clearance would be recovered to a certain degree. However, diluted nasal mucus might have limited role in resolving the nasal symptoms. Therefore, although conservative nasal hydration can also enhance mucociliary clearance, nasal symptoms did not improve significantly with this treatment option. Also, nasal spray and irrigation requires saline and special instruments and should be carried out consistently to control symptoms. In contrast, PRP injections can achieve good results after only 1 injection. After injection, the bulk of the turbinate increases immediately. However, at the next outpatient visit (2 weeks later), there was no difference in inferior turbinate volume and shape. Therefore, the effect of temporary volume increase due to injection is thought to be insignificant. Also, as shown in SNOT-22 subdomain subanalysis results, emotional subdomain was not improved significantly in the patients with PRP injections. The result, not being accompanied by improvements in the emotional subdomain, suggests that the placebo effect was not significant in an aspect.

The constant pain-and discomfort-related symptoms of atrophic rhinitis are not at medically critical status. Therefore, socioeconomic loss has been underestimated. However, it is a socially critical disease for the point of view of the actual patients. Also, as the population is aging, the number of patients showing geriatrics rhinitis is increasing exponentially. Therefore, it is very important to develop proper treatment for atrophic rhinitis including geriatrics rhinitis.

This study aimed to present a new regenerative method for atrophic rhinitis management through autologous PRP injection and used a control group to compare the improvement of symptoms between the PRP treatment group and the conservative treatment group. As atrophic rhinitis is a clinical definition, more studies are required to elicit the mode of action. However, to prevent socioeconomic losses and downgraded quality of life of patients, it is essential to present research results that have the potential for therapeutic outcomes.

However, there were several limitations in this study. The study was designed prospectively and could not have a randomized controlled design because some participants declined the nasal injection procedure. In the aspect of ethics, invasive procedure to the control group for academic purpose should be limited, to protect the patients' rights from being harmed. Thus, the patients were assigned according to their preference, which rendered the findings weaker than those afforded by randomized controlled studies. Also, secondary atrophic rhinitis is caused by deformation and removal of original nasal structures. Taking a biopsy could aggravate atrophic condition. Therefore, in the aspect of ethics, it is deemed impossible to conduct biopsy of the corresponding nasal tissue in the patients with atrophic rhinitis. The results after 6 months of follow-up may not be representative of the long-term results. In addition, the subgroups had relatively small numbers of patients. Therefore, future studies should include a larger number of patients with a randomized controlled design over a longer follow-up period. 


\section{Conclusion}

This study presented a new regenerative method for atrophic rhinitis management through autologous PRP injection. Patients with atrophic rhinitis can improve their nasal symptoms and nasal mucociliary function with PRP injection. Also, younger patients showed better improvements than the older group. However, future randomized controlled studies with a larger number of patients and a longer follow-up period are needed to verify these results.

\section{Statement of Ethics}

This clinical trial was approved by the Institutional Review Board of the Seoul St. Mary's Hospital (KC16CISI0726). The trial was registered in the database of clinical trials (NCT03112330). This study was monitored by the Catholic Institute of Cell Therapy, Catholic Medical Center, Korea. All investigators conducted this study in accordance with the Declaration of Helsinki. Written informed consent was obtained from all patients before recruitment.

\section{Conflict of Interest Statement}

The authors declare that there are no conflicts of interest.

\section{Funding Sources}

This research was supported by the Basic Science Research Program through the National Research Foundation of Korea (NRF) funded by the Ministry of Education (2020R1I1A1A01051844) and the Bio \& Medical Technology Development Program of the NRFfunded by the MinistryofScience\&ICT (2018M3A9E8020856, 2019M3A9H2032424, and 2019M3E5D5064110). The sponsors had no role in the study design, data collection and analysis, decision to publish, or preparation of the manuscript.

\section{Author Contributions}

Do Hyun Kim and Sung Won Kim: study conception and design, acquisition of data, analysis and interpretation of data, drafting the article and revisions, and final approval of the article; Min Hyeong Lee, Eun A. Song, Jaeyoon Lee, and Soo Whan Kim: study conception and design, analysis and interpretation of data, drafting the article and revisions, and final approval of the article.

\section{References}

1 Mishra A, Kawatra R, Gola M. Interventions for atrophic rhinitis. Cochrane Database Syst Rev. 2012 Feb 15;(2):Cd008280.

2 Bist SS, Bisht M, Purohit JP. Primary atrophic rhinitis: a clinical profile, microbiological and radiological study. ISRN Otolaryngol. 2012 Nov 19;2012:404075.

3 Baptist AP, Nyenhuis S. Rhinitis in the elderly. Immunol Allergy Clin North Am. 2016 May;36(2):343-57.

4 Moore EJ, Kern EB. Atrophic rhinitis: a review of 242 cases. Am J Rhinol. $2001 \mathrm{Nov}-$ Dec;15(6):355-61.

5 Marx RE. Platelet-rich plasma: evidence to support its use. J Oral Maxillofac Surg. 2004 Apr;62(4):489-96.

6 Salaheldin AH, Hussein A. Effect of plateletrich plasma on nasal mucociliary clearance after submucous diathermy of inferior turbinate. EJENTAS. 2012 Jul 1;13(2):71-5.
7 Friji MT, Gopalakrishnan S, Verma SK, Parida PK, Mohapatra DP. New regenerative approach to atrophic rhinitis using autologous lipoaspirate transfer and platelet-rich plasma in five patients: our experience. Clin Otolaryngol. 2014 Oct;39(5):289-92.

8 Stewart MG, Witsell DL, Smith TL, Weaver EM, Yueh B, Hannley MT. Development and validation of the Nasal Obstruction Symptom Evaluation (NOSE) scale. Otolaryngologyhead and neck surgery. Otolaryngol Head Neck Surg. 2004 Feb;130(2):157-63.

9 Hopkins C, Gillett S, Slack R, Lund VJ, Browne JP. Psychometric validity of the 22item sinonasal outcome test. Clinical otolaryngology : official journal of ENT-UK. Clin Otolaryngol. 2009 Oct;34(5):447-54.

10 Plaza Valia P, Carrion Valero F, Marin Pardo J, Bautista Rentero D, Gonzalez Monte C. [Saccharin test for the study of mucociliary clearance: reference values for a Spanish population]. Arch Bronconeumol. 2008 Oct; 44(10):540-5.
11 Feng AL, Wesely NC, Hoehle LP, Phillips KM, Yamasaki A, Campbell AP, et al. A validated model for the 22-item sino-nasal outcome test subdomain structure in chronic rhinosinusitis. Int Forum Allergy Rhinol. 2017 Dec;7(12):1140-8.

12 Jaswal A, Jana AK, Sikder B, Nandi TK, Sadhukhan SK, Das A. Novel treatment of atrophic rhinitis: early results. Eur Arch Otorhinolaryngol. 2008 Oct;265(10):1211-7.

13 Pavithran P, Pujary K, Mahesh SG, Parul P, Aziz B. Customised acrylic nasal stents following recanalisation of modified young's procedure. J Laryngol Otol. 2010 Aug;124(8): 864-7.

14 Stavrakas M, Karkos PD, Markou K, Grigoriadis N. Platelet-rich plasma in otolaryngology. J Laryngol Otol. 2016 Dec;130(12):1098102 